\title{
Exposición al benceno en las estaciones de servicio
}

\section{Benzene exposure at service stations}

1 Hugo Fernando Acosta Delgado Universidad Técnica de Manabí hacosta6404@utm.edu.ec

2 Grether Lucía Real Pérez Universidad Técnica de Manabí grether.real@utm.edu.ec
D https://orcid.org/0000-0002-2166-2519

https://orcid.org/0000-0003-4792-6217
Artículo de Investigación Científica y Tecnológica

Enviado: 12/12/2021

Revisado: $27 / 12 / 2021$

Aceptado: 28/01/2022

Publicado:05/02/2022

DOI: https://doi.org/10.33262/ap.v4i1.1.176

\section{$\begin{array}{lll}\text { Cítese: } & \text { estaciones } & \text { https://doi.org/10.33262/ap.v4i1.1.176 }\end{array}$ estaciones de servicio. AlfaPublicaciones, 4(1.1), 485-498.}

Acosta Delgado, H. F., \& Real Pérez, G. L. (2022). Exposición al benceno en las

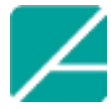

Ciencia

Digital

ALFA PUBLICACIONES, es una Revista Multidisciplinar, Trimestral, que se publicará en soporte electrónico tiene como misión contribuir a la formación de profesionales competentes con visión humanística y crítica que sean capaces de exponer sus resultados investigativos y científicos en la misma medida que se promueva mediante su intervención cambios positivos en la sociedad. https://alfapublicaciones.com

La revista es editada por la Editorial Ciencia Digital (Editorial de prestigio registrada en la Cámara Ecuatoriana de Libro con No de Afiliación 663) www.celibro.org.ec 
Palabras

claves:

combustible, hidrocarburos, energía, riesgos, gasolineras.

\section{Keywords:}

fuel, hydrocarbons, energy, risks, gas stations.

\section{Resumen}

Los combustibles fósiles cuentan con una amplia gama de subproductos, los hidrocarburos son hoy en día una de las mayores fuentes de energía del planeta, desde su explotación se han descubierto múltiples usos y una gran cantidad de subproductos derivados de la destilación de este compuesto, el fraccionamiento de los hidrocarburos han dado subproductos que son ampliamente usados en diferentes áreas de la industria del transporte, tal es el caso de las gasolinas, un subproducto proveniente de la destilación del petróleo crudo, su uso básicamente está enfocado a los vehículos. En diferentes partes del mundo se producen diferentes mezclas de gasolina, con la finalidad de responder a las diferentes necesidades de cada región, sin embargo, su composición consta de diferentes compuestos aromáticos entre esos el benceno, el cual se aborda de manera especial en este artículo por ser uno de los compuestos de la gasolina más perjudiciales a la salud del ser humano. De igual manera se especifican los múltiples riesgos asociados al trabajo que desempeñan los despachadores de las gasolineras, enfocado siempre en la exposición al benceno y otras sustancias, mencionando los equipos de protección personal necesarios para minimizar dichos riesgos.

\section{Abstract}

Fossil fuels have a wide range of by-product uses, the range of hydrocarbons are today one of the largest sources of energy on the planet, since their exploitation multiple and a large number of byproducts derived from the distillation of this compound have been discovered, The fractionation of hydrocarbons has given byproducts that are widely used in different areas of the transportation industry, such is the case of gasoline, a by-product from the distillation of crude oil, its use is basically focused on vehicles. In different parts of the world, different blends of gasoline are produced, in order to respond to the different needs of each region; however, its composition consists of different aromatic compounds, including benzene, which is addressed in a special way in this article by be one of the most harmful gasoline compounds to human health. In the same way, the multiple risks associated with the work carried out by gas station dispatchers are specified, always focused on exposure to benzene and other 
substances, mentioning the personal protective equipment necessary to minimize said risks.

\section{Introducción}

Las estaciones de servicio o gasolineras son centros en los que se desarrollan diversos tipos de trabajo, no solo de despacha combustible a los vehículos, sino que se realizan trabajos mecánicos, autolavado, venta y distribución de repuestos y lubricantes para diferentes tipos de vehículos, venta de comida, entre otros, por lo cual hablar de los riesgos que pueden afectar a los trabajadores de estos centros laborales implica realizar una identificación y valoración de cada puesto de trabajo y en función de cada proceso de trabajo ponderar los riesgos asociados, para el caso de este trabajo de investigación se aborda el tema de la exposición de los trabajadores o despachadores de gasolina o combustible en las estaciones de servicio, con especial atención a la exposición del benceno, el cual forma parte del grupo de los BTEX, son compuestos aromáticos que están naturalmente en los crudos de los cuales provienen la gasolina y el diesel, también se adicionan a la gasolina para aumentar su octanaje y se emiten a la atmósfera por la combustión de dichos combustible. Los compuestos aromáticos presentan propiedades tóxicas, cancerígenas, mutagénicas y anestésicas. El benceno es un componente que tiene gran importancia toxicológico para el hombre por sus efectos cancerígenos, puede generar efectos en la salud como leucemia en trabajadores expuestos (Dehghani et al., 2018).

Estos compuestos presentan gran porcentaje de toxicidad a la salud, en grandes concentraciones y tiempos de exposición prolongados pueden causar el desarrollo de carcinomas, anemias entre otras enfermedades degenerativas crónicas a las personas que están expuestas a estos compuestos, más aun a los trabajadores de las gasolineras, quienes a diario cumplen la función de manipulación de máquinas despachadoras de combustibles, y son el personal que se encuentra en la primera línea de acción al momento de abrir la tapa del tanque de almacenaje de combustible de cada vehículo y es quien acciona la maquina despachadora y manipula a manguera con la cual se llena cada tanque de cada vehículo, esta acción, que pudiese llegar a parecer simple, reviste un gran riesgo para esos trabajadores, puesto que es allí cuando los gases tóxicos llegan a causar daño a la salud de los trabajadores.

Adicionalmente, se conocerá que no solo los despachadores están en riesgo latente, sino que los otros trabajadores de la estación de servicio pueden resultar afectados; por otra parte, se mencionan los diferentes riesgos tanto físicos como mecánicos y eléctricos adicionales a los que los trabajadores se exponen. 
La presente investigación trata de exponer como el benceno, logra penetrar en el cuerpo humano y provocar daños irreparables al organismo. Por medio de una investigación bibliográfica.

\section{Metodología}

La presente investigación se presenta bajo la metodología de revisión bibliográfica, la cual tiene como propósito realizar consultas de diferentes autores con la finalidad de generar una serie de conclusiones y discusión de los resultados, la revisión de la literatura implica detectar, consultar y obtener la bibliografía (referencias) y otros materiales que sean útiles para los propósitos del estudio, de donde se tiene que extraer y recopilar la información relevante y necesaria para enmarcar nuestro problema de investigación (Vásquez, 2020).

Por otro lado, es de tipo documental, ya que el investigador realiza una búsqueda de información de segunda mano, cuando busca y elige aquella información que ya está documentada: registrada, recopilada y clasificada; información que puede estar en forma de escritura, voz, imagen, sonido, símbolos gráficos, tablas o cuadros estadísticos, mapa, dibujo, escultura, etc. (Vásquez, 2020).

La presente investigación se realizó bajo la revisión de diferentes artículos, libros, trabajos de grado, proyectos, revistas científicas y fuentes verificables que garantizan la fiabilidad de los conceptos y análisis que se presentan.

\section{Resultados y Discusión}

Luego del descubrimiento del Petróleo, el mundo entero pudo constatar los grandes beneficios del uso de este nuevo recurso que proporcionaba la madre tierra, solo fueron necesarios los respectivos estudios y análisis de parte de los científicos para verificar que ese elemento encontrado en grandes cantidades debajo de la tierra, no sería más que la mayor fuente de energía con que contaría el planeta y las diferentes empresas y negocios para realizar y/o complementar las múltiples invenciones que para la época salían a la luz pero que necesitaban algún tipo de combustible que hiciera posible su funcionamiento. Según Vargas (2020):

"El primer pozo petrolero se perfora en el año de 1859 en Oíl Creek, Pensilvania, a la cabeza de Edwin Drake, un hombre que trabajaba como conductor de ferrocarriles y convertido en accionista y presidente de Seneca Oíl Company por James Townsend, un patrocinador de dicha compañía. El 27 de agosto de 1859, con una profundidad perforada de 69 pies, se evidenció fluido en la parte superior del pozo, era petróleo. Sin darse cuenta, Drake había perforado en un lugar donde se podía encontrar crudo a una profundidad muy baja”. 
Esto marcaria la nueva era de la energía en el planeta, los múltiples usos a los que posteriormente fue implementado el petróleo representarían un gran avance en las tecnologías que para ese entonces el mundo estaría por conocer. Sin embargo, este hidrocarburo en su estado puro no tiene muchos usos, por lo que fue necesario realizar diferentes separaciones químicas para procesarlo y de esta manera lograr un mejor aprovechamiento de dicho recurso, seguidamente se presenta las diferentes etapas por las que pasa el petróleo en la búsqueda de sus diferentes subproductos.

\section{Tabla 1}

Etapas de la Cadena de Valor de Hidrocarburos

\begin{tabular}{|c|l|l|}
\hline ETAPA & \multicolumn{1}{|c|}{ ACTIVIDADES } & \multicolumn{1}{c|}{ DESCRIPCIÓN } \\
\hline EXTRACCIÓN & $\begin{array}{l}\text { Exploración, perforación y } \\
\text { producción }\end{array}$ & $\begin{array}{l}\text { Etapa desde la búsqueda de crudo } \\
\text { hasta la producción del mismo }\end{array}$ \\
\hline TRANSPORTE & $\begin{array}{l}\text { Transporte en oleoductos y y } \\
\text { gasoductos }\end{array}$ & $\begin{array}{l}\text { Traslado desde el pozo hacia las } \\
\text { plantas procesadoras }\end{array}$ \\
\hline PROCESAMIENTO & Separación y refinación & $\begin{array}{l}\text { Separación de hidrocarburos y } \\
\text { refinación para obtener productos } \\
\text { de uso final }\end{array}$ \\
\hline COMERCIALIZACION & Distribución y ventas & $\begin{array}{l}\text { Se distribuyen los subproductos } \\
\text { obtenidos en el mercado interno o } \\
\text { se exporta }\end{array}$ \\
\hline
\end{tabular}

Fuente: Vargas (2020)

De acuerdo a la información reflejada en la Tabla 1, se puede verificar que son cuatro etapas básicas por las que el petróleo crudo pasa para extraer sus variados subproductos, pero es en la tercera etapa en la cual se obtiene los diversos subproductos luego de la refinación y destilación del petróleo, tal es el caso de la gasolina, la cual es un producto obtenido en la destilación fraccionada del petróleo crudo, esto significa que depende del crudo que se destile es una fracción única o una mezcla de diversas fracciones, está formada de una mezcla de hidrocarburos de peso molecular no muy elevado debe ser volátil, par que queme fácilmente y para mejorar el arranque en frio, pero no tanto como para formar demasiado vapor con el tiempo caluros, esto de acuerdo con (CCT, 2011) mencionado por (Pérez, 2018). 


\section{Figura 1}

Destilación del petróleo

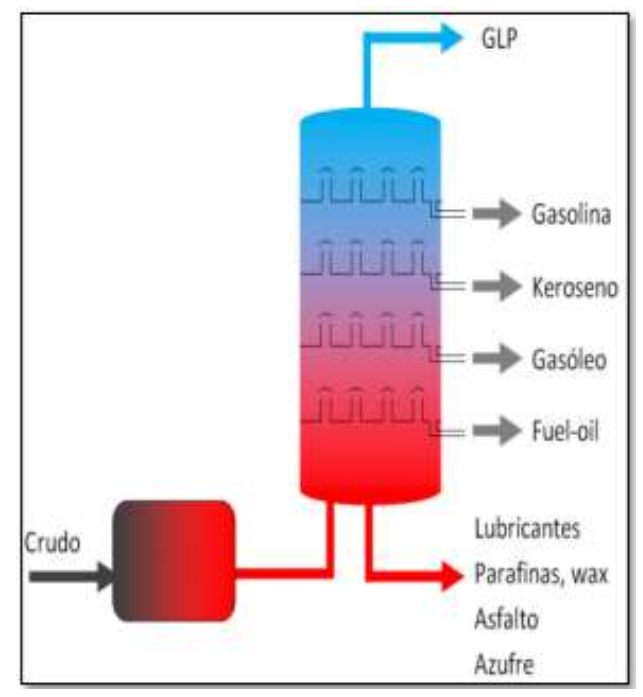

Fuente: Legrand (2015)

De acuerdo a lo descrito por Boluda et al. (2019) las gasolinas, al igual que otros combustibles de automoción, deben cumplir con las especificaciones de los fabricantes de vehículos, cuyas orientaciones se recogen en la Carta Mundial de Combustibles y con las normativas vigentes de cada país. Dichas normativas han evolucionado a lo largo de las últimas décadas, aumentando notablemente los niveles de exigencia, con el objeto de proteger la salud de la población y el medio ambiente, estos combustibles no solo han despertado gran utilidad en casi todas las actividades de la vida cotidiana desde que fueron descubiertos, sino que también han sido objeto de muchos juicios debido a los grandes y nocivos impactos en el medio ambiente así como en la salud de la población mundial en general, desde la extracción hasta la disposición final de dichos combustibles, es notoria y grave los daños ocasionados a la salud del planeta y del ser humano, los gases emitidos por la combustión de combustibles fósiles han llevado al planeta tierra al borde de una de las crisis climáticas más preocupantes en toda la historia del planeta, nunca antes se habían manejado tantos cambios en el clima y condiciones generales del planeta como ahora, y todo se debe a los efectos contaminantes ocasionados en su gran mayoría por los combustibles fósiles y su uso indiscriminado y sin un control aparente.

En este caso en particular, se tocará el tema de los diferentes riesgos a los que se encuentra expuesto un trabajador en una estación de servicio o gasolinera, lo cual reviste de gran importancia ya que como se mencionó anteriormente la gasolina es un derivado del procesamiento del petróleo, aparte de que su composición físico química supone un fuerte contaminante al ambiente y sobre todo a la salud del ser humano. Según Santiago (2019): 
La gasolina, una mezcla compleja que además de otros compuestos, contiene hidrocarburos aromáticos, entre los cuales, la hoja de seguridad MSDS (Material Safety Data Sheet), destaca el benceno, compuestos con el porcentaje de concentración más relevante. Este hidrocarburo aromático es un disolvente orgánico altamente inflamable y catalogado carcinógeno por la organización mundial de la salud (OMS), dentro de los grupos cancerígenos está clasificado en la categoría tres por las Naciones Unidas (ONU), en el grupo A1 por la conferencia Americana de Higienistas Industriales Gubernamentales (ACGIH) y en la clase 1 por el Centro Internacional de Investigaciones sobre el Cáncer (IARC), por consiguiente, el uso de esta sustancia química es restringido, y en cuanto a la manipulación de la sustancia, internacionalmente se han establecido limites ocupacionales con el propósito de velar por el bienestar de los trabajadores que tienen contacto directo y/o indirecto con este compuesto de la gasolina.

Según investigaciones al respecto de las exposiciones de compuestos de las gasolinas, Dehghani et al. (2018) determinan que el benceno es el compuesto orgánico volátil que predomina en estos puntos, alcanzando concentraciones de $26,15 \pm 17,65 \mu \mathrm{g} / \mathrm{m} 3$ en las mañanas y $34,44 \pm 15,63 \mu \mathrm{g} / \mathrm{m} 3$ durante las tardes. Por otra parte, el benceno supera la concentración de tolueno en proporciones que oscilan entre 2,02 y 3,07. El riesgo de inhalación de por vida (LTCR) para el benceno en la mañana y en la noche fue de 1,96 $\times 10-4$ y 2,49 ×10-4, respectivamente; que evidentemente supera los límites propuestos por la Organización Mundial de la Salud. Por otro lado, Chen, et al. (2016) han determinado que el benceno tiene repercusiones más graves en cuanto al riesgo de cáncer, puesto que el exceso de riesgo de cáncer de por vida por la exposición al benceno se estimó en $2,22 \times 10-5,2,43 \times 10-6$ y 2,56 × 10-7 para empleados de estaciones de servicio completo, empleados de estaciones de autoservicio y usuarios de estaciones de autoservicio que se supone que usan la estación una vez por semana, respectivamente. Esto evidencia las graves consecuencias que trae a la salud de los trabajadores la exposición continua a los compuestos y gases emitidos por las gasolinas en las estaciones de servicio o gasolineras, este tipo de exposición no solo desencadena en enfermedades del sistema respiratorio, sino que atenta a nivel celular provocando el desarrollo de enfermedades asociadas al cáncer.

De igual manera se puede evidenciar que existe un riesgo mayor de provocar enfermedades por exposición en las gasolineras por género, en este sentido Moro et al. (2017) exponen que se observa un notable aumento de mujeres trabajadoras de las estaciones de servicio o gasolineras en el mundo y tras la evaluación de parámetros biológicos como niveles urinarios de ácido trans, trans-mucónico, alteraciones genéticas y hematológicas bien conocidas por su relación con los efectos no cancerosos en los sistemas hepáticos, renales e inmunitarios en personas expuestas al benceno; se 
determinaron posibles efectos del género sobre las consecuencias de la exposición ocupacional al benceno, particularmente en los parámetros hematológicos y en los niveles de ácido trans, trans-mucónico, lo que sugiere que las alteraciones en ciertos parámetros a nivel de test médicos realizados en diferentes estudios a mujeres que trabajan en gasolineras, expone que el género puede influir en la aparición o no de patologías asociadas a los carcinomas.

De igual manera, para Jacome (2021):

La exposición prolongada al benceno afecta principalmente a la sangre disminuyendo los glóbulos rojos, conllevando a una anemia, afecta el sistema inmunitario dejándolo susceptible de contraer infecciones. Existen casos en los cuales las mujeres, al estar expuestas a niveles altos de benceno por varios meses presentan menstruaciones irregulares, disminución del tamaño de los ovarios, entre otros síntomas.

A continuación, se puede observar, en la figura 2, la forma como se expone el trabajador a los gases tóxicos en la estación de servicio.

\section{Figura 2}

\section{Estación de servicio automotriz, fuente de liberación crónica de combustible}

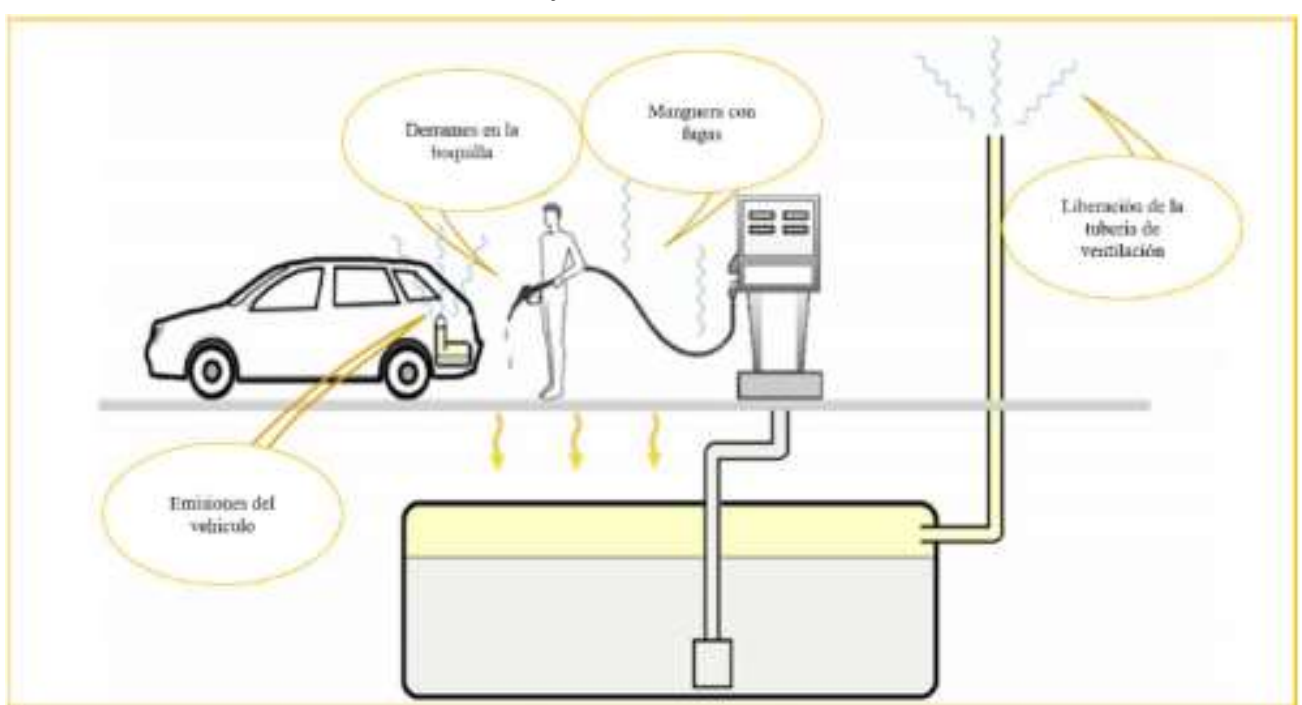

Fuente: Hilpert et al. (2015)

Desde el punto de vista toxicológico, el benceno es considerado el contaminante más importante, se ha demostrado en varios estudios que afecta a los sistemas nervioso, linfático, hematopoyético, hepático, renal, además de ser considerado como cancerígeno para el hombre. Las afectaciones en la salud de los despachadores están dadas por la concentración de los vapores, su inhalación, ingestión o contacto con la piel y mucosas, causando efectos agudos y crónico (Romero et al., 2017). La persona al estar expuestas a 
concentraciones de 1ppm de benceno en el aire presentara disminución de glóbulos blancos, plaquetas, mutación de ADN, anormalidades cromosómicas y daño oxidativo. La concentración de benceno en sangre de $2,1 \mathrm{mg} / \mathrm{L}$ provocan depresión del sistema nervioso y concentraciones de benceno en el aire de $65 \mathrm{mg} / \mathrm{L}$ provocan consecuencias letales en el período de 5 a 10 minutos de exposición (Santana et al., 2020).

Según Zubizarreta et al. (2018):

El benceno penetra en el organismo principalmente por inhalación, aunque la absorción cutánea es también posible. Después de su absorción, el benceno es eliminado inalterado en la orina (menos del 1\%) y en el aire expirado (10 a 50\% según la actividad física y la importancia del tejido adiposo); el resto es bio transformado. La mayor parte del benceno absorbido es metabolizado, básicamente en el hígado y la médula ósea, por oxidación a fenol, quinol y catecol, que se excretan en la orina en forma de sulfatos y glucuronatos. La metabolización y eliminación del benceno es rápida. La excreción de los metabolitos se completa generalmente dentro de las 24-48 horas después de una exposición única, lo que representa una vida media biológica inferior a las 12 horas. Sin embargo, los tejidos adiposos pueden retener una pequeña cantidad de benceno durante varios días después del final de la exposición.

A continuación, se muestra una imagen la cual describe el metabolismo del Benceno en el cuerpo humano.

\section{Figura 3}

\section{Metabolismo del benceno}

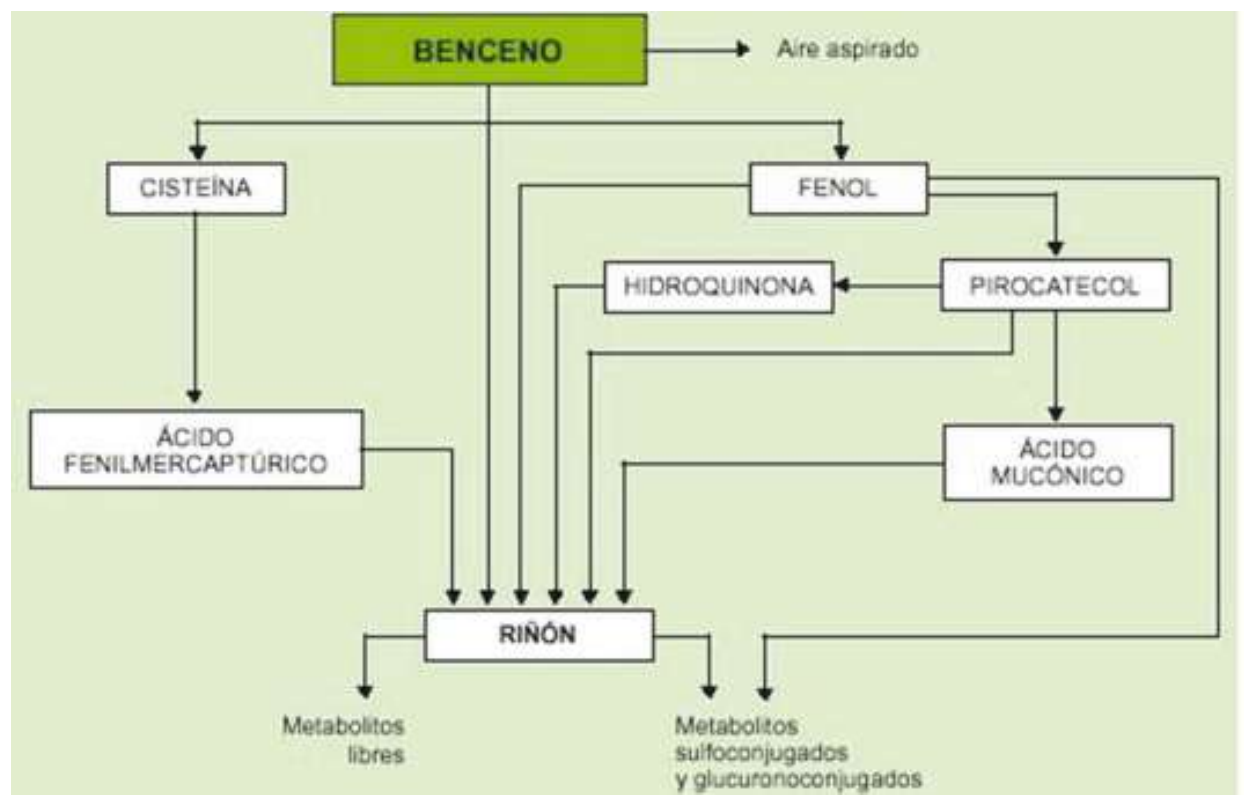

Fuente: Zubizarreta et al. (2018) 
Los síntomas a causa de la intoxicación con este compuesto de la gasolina varían de acuerdo al tiempo de exposición y a la concentración del compuesto; sin embargo, van desde dolores de cabeza, náuseas, desorientación, irritación de la mucosa ocular y garganta, así como palpitaciones y dificultad para respirar. Cabe destacar, que en mucho de los casos los despachadores de gasolina realizan sus labores en turnos de 8 horas y muchas veces sin ningún tipo de equipo de protección personal, lo que supondría un mayor riesgo de exposición y de efectos secundarios a la salud del trabajador.

\section{Figura 4}

\section{Perfil de toxicidad multisistémica}

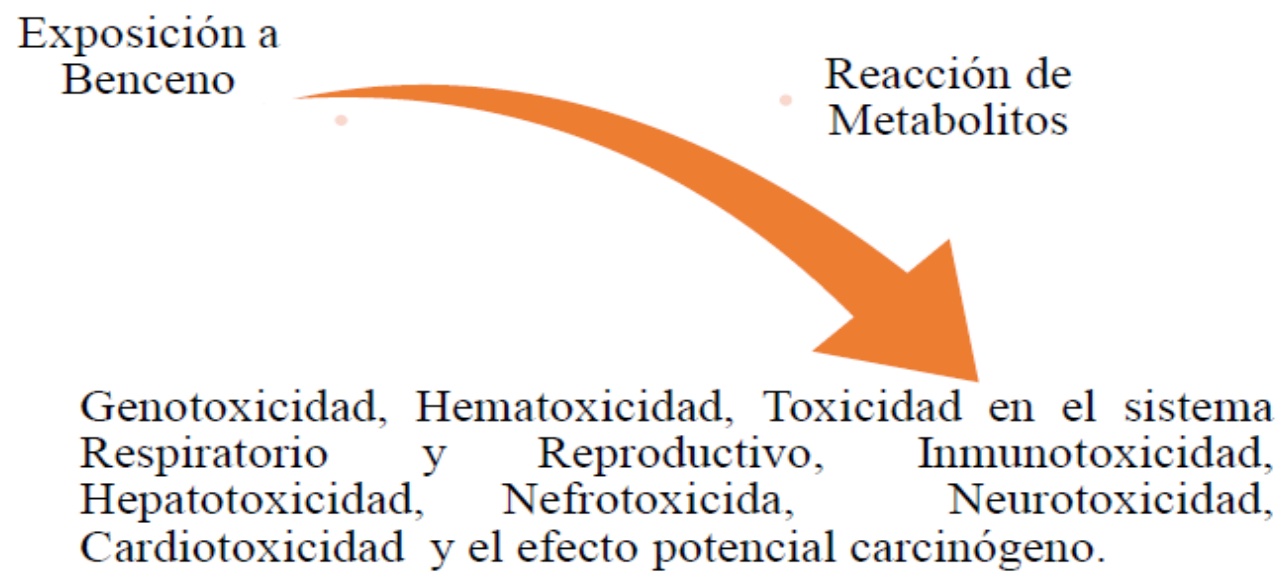

Fuente: Santiago (2019)

Con respecto a los equipos de protección personal, el primer elemento a valorar son las máscaras respiratorias. La protección de la piel, segunda vía principal de exposición, puede lograrse mediante el uso de prendas protectoras como guantes, protectores y máscaras faciales y mandiles. Además, deberán utilizarse gafas protectoras en caso de que exista riesgo de recibir salpicaduras en los ojos de estas sustancias. Los trabajadores no deben utilizar lentes de contacto cuando trabajen en áreas con riesgo de exposición, especialmente de la cara y los ojos, ya que las lentes de contacto, si no se quitan inmediatamente, pueden potenciar el efecto nocivo de estas sustancias y hacer que los lavados oculares sean menos eficaces (Zubizarreta et al., 2018).

La exposición al benceno en gasolineras es una problemática estudiada en varios países del mundo, que demuestran mediante resultados concluyentes que el benceno afecta significativamente la salud y seguridad de trabajadores permanentes, ocasionales y a usuarios de las estaciones de servicio. Sin embargo, aún no se aplican estrategias sanitarias globales para precautelar la salud de los trabajadores expuestos a este compuesto volátil. A nivel mundial se reportan deficiencias en el control, monitoreo y seguimiento de las condiciones de seguridad y salud de los trabajadores de estaciones de servicio de distribución y venta de combustibles, por lo tanto, los riesgos asociados con 
la exposición de compuestos orgánicos volátiles como el benceno aumentan significativamente (Santana et al., 2020).

En líneas generales, los trabajadores de las gasolineras están expuestos a los siguientes riesgos: caídas al mismo nivel, incendios y explosiones, sobreesfuerzos por posturas inadecuadas y carga de objetos, atropellos o golpes con vehículos, contactos térmicos, exposición a temperaturas y condiciones ambientales cambiantes y extremas, como los ruidos, viento, lluvia y frío, Exposiciones a inhalaciones de gases de la combustión como monóxido de carbono, óxidos de azufre y otros componentes de los hidrocarburos como el benceno, que es cancerígeno, Dermatitis por contacto con los combustibles, lubricantes (sobre todo los usados) y otros agresivos, contactos eléctricos e incluso están expuestos a la violencia por riesgos de robos o hurtos (Agramonte, 2018).

De acuerdo a lo anteriormente descrito, los riesgos a los cuales los trabajadores de las estaciones de servicio o gasolineras son muy variados y de intensidad diversa, ya que no todos son de una misma naturaleza, como en casi todos los centros de trabajo los trabajadores están expuestos a riesgos como caídas del mismo y distinto nivel, choques eléctricos, posturas inadecuadas y forzadas, pero en particular se añaden riesgos graves como la exposición a inhalación de gases extremadamente dañinos a la salud, como el benceno, uno de los componentes de la gasolina, esto sin contar con las exposiciones cutáneas a las mismas sustancias, aumentando el riesgo a sufrir enfermedades de las mucosas oculares y garganta, así como en el peor de los casos a desarrollar carcinomas, e incluso a sufrir daños a los sistemas nervioso y respiratorio, lo cual suponen para los trabajadores grandes consideraciones a tomar en cuenta al momento de decidir trabajar en estos centros laborales.

De igual manera, los dueños de este tipo de establecimientos deben contar con planes de higiene y seguridad ocupacional que permita mitigar o minimizar estos riesgos, si bien es cierto que resulta cuesta arriba afrontar este tipo de riesgos, también lo es que, con una capacitación constante y oportuna, así como la dotación y uso correcto de equipos de protección personal se puede minimizar los efectos negativos sobre la salud de los empleados. Por otra parte, los diferentes estudios que se han realizado sobre los niveles permisibles en el mundo sobre las exposiciones de estos gases se pueden evidenciar que las empresas extractoras y procesadoras de hidrocarburos deben cumplir con las perisologías exigidas a nivel mundial sobre la toxicidad de los hidrocarburos y sus derivados, así como garantizar a sus trabajadores una manipulación correcta, así como el transporte y almacenaje seguro de dichos elementos.

Aún queda mucho por hacer en materia de riesgos a causa de los hidrocarburos, no solo a nivel ambiental, que ya bastantes consecuencias negativas han traído al planeta sino también a la salud pública, día a día no solo los trabajadores de las estaciones de servicio están en riesgo latente y constante de sufrir consecuencias graves a su salud a causa de 
las emisiones de gases provenientes de combustibles fósiles, sino también a la población en general, según la OIT una de las más frecuentes y recurrentes enfermedades en la población mundial son las respiratorias, las cuales están relacionadas directamente por la acción de los vapores tóxicos disueltos en el ambiente.

\section{Conclusiones}

- Por medio de la destilación del petróleo se obtiene los diferentes subproductos dentro del cual se encuentra la gasolina, uno de los combustibles que se despachan o se comercializan en una estación de servicio o gasolinera, su proceso comprende diferentes métodos y dependiendo del uso o destino se agregan o no diferentes aditivos, los cuales le dan sus características particulares.

- La gasolina la componen el grupo de los BEXT (benceno, tolueno y xileno), compuestos aromáticos entre los que destacan el benceno, el compuesto que presenta más toxicidad y por ende el que más representa riesgo a los trabajadores de las gasolineras. Se pudo conocer que en bajas concentraciones y poco tiempo de exposición puede ocasionar irritación en los ojos y garganta, y en los casos más severos de exposición pueden a llegar a afectar el sistema nervioso central, sistema respiratorio e incluso llegar a desarrollar carcinoma.

- En muchos de los casos los trabajadores de estos centros no cuentan con los equipos de protección personal necesarios y adecuados para evitar o al menos disminuir los riesgos presentes, en muchos de los casos porque sus empleadores no realizan la respectiva dotación o simplemente desconocen cuáles son los equipos realmente necesarios, y en otros casos los trabajadores por negligencia dejan de usarlos.

- Los organismos internacionales encargados de las regulaciones de las emisiones de estos gases tóxicos aún deben realizar muchos cambios en ser más estrictos para evitar la gran cantidad de enfermedades y efectos adversos al ambiente que han provocado en el planeta entero y a la población en general.

\section{Referencias Bibliográficas}

Agramonte, J. (2018). Diseño de un sistema de gestión de la seguridad y salud en el trabajo, bajo los requisitos de la ley 29783, en la empresa gasolineras Piura s.r.l. Piura: para optar el título profesional de ingeniero ambiental de la universidad alas peruanas.

Boluda, C., Macias, M., \& González, J. (2019). La complejidad química de las gasolinas de automoción. Ciencia, Ingenierías y Aplicaciones, 51-79. 
Chen, X., Feng, L., Luo, H., \& Cheng, H. (2016). Health risk equations and risk assessment of airborne benzene homologues exposure to drivers and passengers in taxi cabins. Environmental Science and Pollution Research, 4797-4811.

Dehghani, M., Fazlzadeh, M., Sorooshian, A., Tabatabaee, H., Miri, M., Baghani, A., \& Rashidi, M. (2018). Characteristics and health effects of BTEX in a hot spot for urban $c b$.

Hilpert, M., Mara, B., Dria, A., Ni, J., Rule, A., \& Nachman, K. (2015). Hydrocarbon release during fuel storage and transfer at gas stations: environmental health effects. Current environmental health reports, 412-422.

Jacome, A. (2021). Efectos en la salud de los despachadores de gasolineras por exposición a hidrocarburos en Adecamor. Uniandes.

Legrand, M. (2015). Fuentes de Energía Capitulo 4. Petróleo, combustibles líquidos y gaseosos. Madrid: Leganes.

Moro, A., Brucker, N., Charao, M., Baierle, M., Sauer, E., Goethel, G., García, S. (2017). Biomonitoreo de Empleados de Gasolineras Expuestos a Benceno. Mutation Research/Genetic Toxicology and Environmental Mutagenesis, 1-9.

Pérez, D. (2018). Estudio de Emisiones Contaminantes Utilizando Combustibles Locales. Innova Research, 23-34.

Romero, G., Medina, A., Marrero, S., Moran, A., \& Montolla, M. (2017). Evaluación de la Exposición a Benceno en Trabajadores de Diferentes Áreas Laborales. Salud Uninorte, 33 .

Santana, M., Torrens, M., Santana, L., \& García, E. (2020). Enfermedades ocupacionales por exposición a benceno en trabajadores de gasolineras. Revista San Gregorio.

Santiago, S. (2019). Exposición a Benceno en Estaciones de Gasolina. Bucaramanga: proyecto de grado para optar por titulo profesional.

Vargas, L. (2020). Impactos ambientales de la producción petrolera en Colombia y su relación con la innovación tecnológica en los últimos 15 años. Bogotá: Monografía título de especialista.

Vásquez, W. (2020). Metodología de la Investigación. Manual del Estudiante. Universidad San Martin de Porres.

Zubizarreta, A., Martínez, J., Rivas, P., Gómez, S., \& Sanz, A. (2018). Revisión de la literatura sobre efectos nocivos de la exposición laboral a hidrocarburos en trabajadores en ambiente externo. Medicina y Seguridad del Trabajo, 271-94. 
El artículo que se publica es de exclusiva responsabilidad de los autores y no necesariamente reflejan el pensamiento de la Revista Alfa Publicaciones.

\section{Ciencia}

El artículo queda en propiedad de la revista y, por tanto, su publicación parcial y/o total en otro medio tiene que ser autorizado por el director de la Revista Alfa Publicaciones.
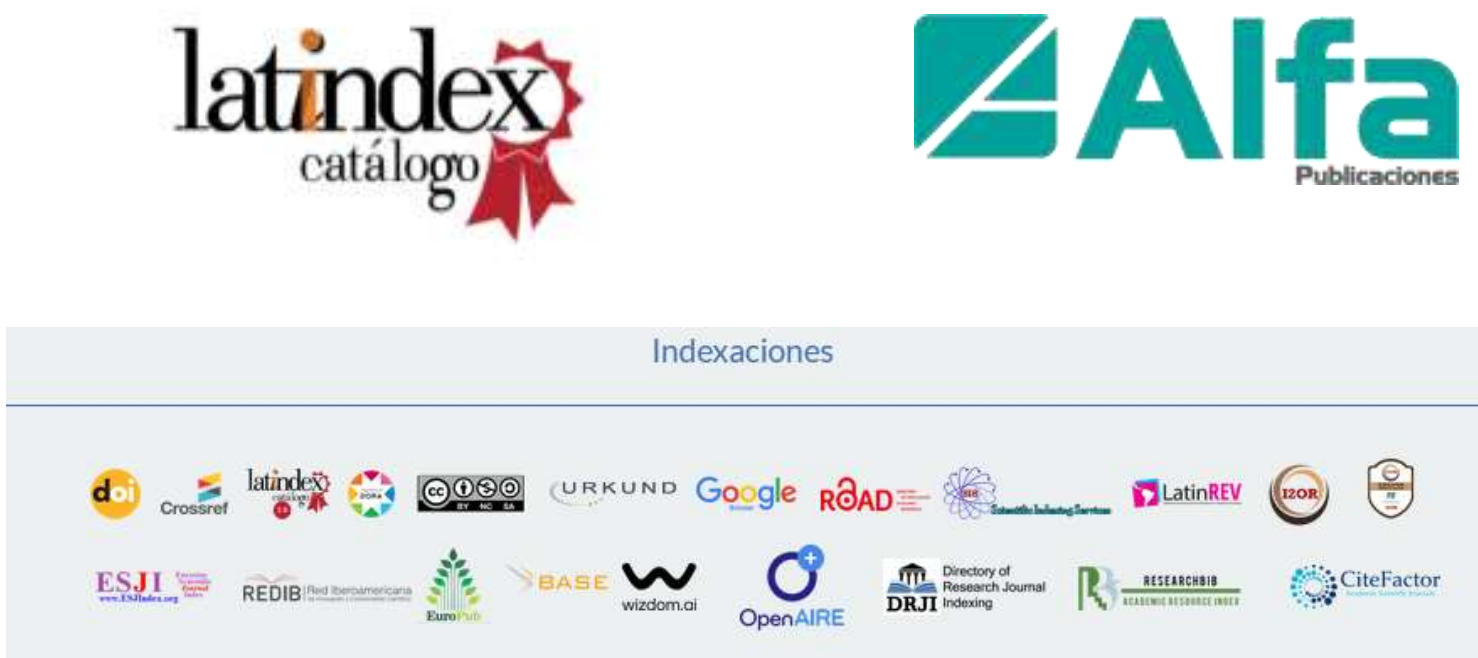